\title{
Modelos de crescimento kaleckianos: uma apreciação
}

\section{MÁRIO AUGUSTO BERTELLA*}

Kaleckian economic growth models: an analysis. The purpose of this article is to survey the main papers that founded a kaleckian approach of the economic growth. It presents a certain moment of the historical evolution of the non-neoclassical macrodynamics. This analysis can be understood under the political economy tradition in putting together social classes (capitalists and workers), distributive conflict and the relationship between distribution and accumulation.

Key-words: growth; distribution; capacity utilization

JEL classification: E12.

\section{INTRODUÇÃO}

O presente trabalho tem o objetivo de examinar os principais modelos que inauguraram o enfoque de inspiração kaleckiana ${ }^{1}$ do crescimento econômico. Procurar-se-á focalizar um determinado momento da evolução histórica da macrodinâmica pós-keynesiana. Esta abordagem insere-se na tradição da economia política ao incorporar a distinção entre as classes sociais (capitalistas e trabalhadores), o conflito distributivo e a relação de causalidade recíproca entre distribuição e acumulação. Tais modelos foram originalmente desenvolvidos de forma independente por Rowthorn (1982) e Dutt (1984).

Em relação aos modelos de primeira geração ou neokeynesianos de Kaldor (1956) e Robinson (1956, 1962), há pelo menos duas grandes diferen-

\footnotetext{
* Professor-Doutor da Faculdade de Filosofia, Ciências e Letras de Araraquara (Fclar/UNESP). O autor agradece os comentários do professor Gilberto T. Lima, eximindo-o dos erros remanescentes. Email: mabertella@yahoo.com.br. Submetido: julho, 2004; aceito: novembro, 2005.

${ }^{1}$ Também denominados modelos estruturalistas ou pós-keynesianos de segunda geração. Doravante, esses termos serão usados indistintamente.
} 
ças. ${ }^{2}$ Em primeiro lugar, os modelos neokeynesianos estão situados em um ambiente de concorrência perfeita, no qual os agentes econômicos são tomadores de preços. Nos modelos atuais, dada a influência kaleckiana, os agentes não são tomadores de preços, mas fazem parte de um ambiente oligopolístico. A segunda grande diferença reside em que, nos modelos de Cambridge, implicitamente, assume-se que a economia opere à plena capacidade ou que o grau de utilização da capacidade é fixo em um dado nível normal, ao passo que, nos modelos de segunda geração, o grau de utilização é endógeno e não é igual a um valor normal, mesmo no longo prazo. Como resultado dessas hipóteses, obtém-se, nos modelos neokeynesianos, uma relação inversa entre taxa de lucro e taxa de acumulação de capital, de um lado, e salário real, de outro. Por outro lado, os modelos à la Kalecki-Steindl podem apresentar uma relação positiva entre salário real, taxa de lucro e acumulação no equilíbrio de longo prazo. ${ }^{3}$

Existem, pelo menos, quatro características nos modelos atuais que devem ser observadas. Em primeiro lugar, os preços relacionados com os custos diretos são influenciados por uma série de fatores freqüentemente resumidos sob o título "grau de monopólio", sendo que o nível de demanda agregada exerce pouca influência sobre o nível de preços. Em segundo, os custos marginais são considerados constantes até alcançar a plena utilização da capacidade instalada. Em terceiro, contrastando com a hipótese dos autores de Cambridge, como Kaldor e Robinson, assume-se que o grau de utilização da capacidade seja inferior ‘a unidade ou diferente de algum valor "normal". Finalmente, o quarto aspecto, e talvez o mais importante, refere-se à função investimento. Esta é dependente não apenas da taxa de lucro, ${ }^{4}$ como nos modelos neokeynesianos, mas também do grau de utilização da capacidade, como defendido por Steindl (1952).

O restante do artigo está organizado como segue: na próxima seção, faremos uma apresentação do modelo canônico; na seção seguinte, a crítica de Bhaduri e Marglin e, em seguida, mostraremos os resultados obtidos quando da abertura da economia ao comércio internacional; a última seção apresenta algumas considerações finais.

\footnotetext{
${ }^{2}$ Os modelos de crescimento de primeira geração surgem tendo como motivação o modelo de Harrod (1939). Kaldor e Robinson atacam Harrod por sua falha em lidar explicitamente com a taxa de lucro e a distribuição de renda. O fundamento dessas críticas baseia-se na rejeição do fio da navalha, isto é, da existência de apenas uma única taxa garantida. Para uma discussão a respeito, vide Bertella (2000).

${ }^{3}$ Para algumas formulações não-lineares recentes na linha desses modelos pós-keynesianos, vide Lima (1998, 1999, 2000). Ver, em particular, Lima (1998), em que a utilização de uma função investimento não-linear permite derivar ambos os resultados dos modelos de $1^{\mathrm{a}} \mathrm{e} 2^{\mathrm{a}}$ geração, mesmo abaixo da plena ocupação da capacidade.

${ }^{4}$ Posteriormente, comentaremos a contribuição de Bhaduri e Marglin (1990) no que concerne à taxa de lucro como argumento da função investimento.
} 


\section{O MODELO CANÔNICO}

As pressuposições básicas do modelo ${ }^{5}$ a serem descritas abaixo são as seguintes:

a. A economia é fechada e produz apenas um bem, que pode ser usado para consumo ou investimento;

b. A tecnologia apresenta coeficientes fixos entre trabalho e capital, e retornos constantes de escala;

c. Não há governo;

d. O lado monetário é ignorado;

e. Todas as empresas são iguais.

Dadas as pressuposições, podemos examinar as equações de preço e produção. A produção pode ser consumida ou investida, de maneira que

$X=C L+g K$,

em que $X$ corresponde ao produto, é o volume de emprego; $K$, ao estoque de capital; $C$, ao consumo por trabalhador empregado; e $g$, à taxa de acumulação de capital.

Dividindo por $X$ e denotando a relação trabalho-produto pelo coeficiente fixo, obtemos

(1) $1=C a_{0}+g\left(\frac{K}{X}\right)$,

que é a equação de produção.

Em relação à equação de preços, temos que parte se destina aos salários e parte aos lucros, assim:

$$
P=w a_{0}+r P\left(\frac{K}{X}\right)
$$

em que $P$ corresponde ao preço, $w$ é o salário nominal por trabalhador, $r$ a taxa de lucro e $r P K$ é o lucro total. Dividindo-se por $P$, obtemos a equação de preços:

(2) $1=V a_{0}+r\left(\frac{K}{X}\right)$,

em que $V$ é o salário real $w / P$.

\footnotetext{
${ }^{5}$ A descrição baseia-se em Dutt (1984, 1987 e 1990). A diferença desse modelo em relação ao de Rowthorn (1982) é que este último trabalha com o fator trabalho composto de mão-de-obra variável e fixa (overhead labour), o que complica um pouco a resolução do modelo, embora os resultados sejam precisamente os mesmos de Dutt, que trabalha apenas com trabalho variável.
} 
Se $a_{1}$ corresponde à relação capital-produto fixa por razões tecnológicas, encontramos:

$$
\frac{K}{X} \geq a_{1},
$$

em que a igualdade representa a plena utilização da capacidade produtiva e valores maiores que $a_{1}$ correspondem a excesso de capacidade. No primeiro caso, em que $K / X=a_{1}$, temos que as equações (1) e (2) transformam-se em:

$$
\begin{array}{ll}
\text { (1') } & 1=C a_{0}+g a_{1} \\
\text { (2') }^{\prime} & 1=V a_{0}+r a_{1}
\end{array}
$$

Assim, se a economia opera à plena capacidade, podemos trabalhar com as equações (1') e (2') e denominá-las fronteira consumo-crescimento potencial e fronteira salário-lucro potencial, respectivamente. Qualquer combinação de pontos $(g, C)$ e $(r, V)$, situada na fronteira potencial, implica que a economia opere com plena utilização da capacidade produtiva. Para graus de utilização da capacidade inferiores à plena, i. e., para $K / X>a_{1}$, devem ser utilizadas as equações (1) e (2). A representação gráfica dessas equações encontra-se abaixo.

Figura 1: Fronteira consumo-crescimento potencial

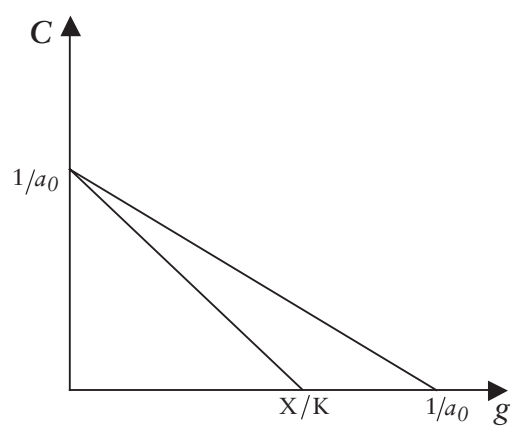

Figura 2: Fronteira salário-lucro potencial

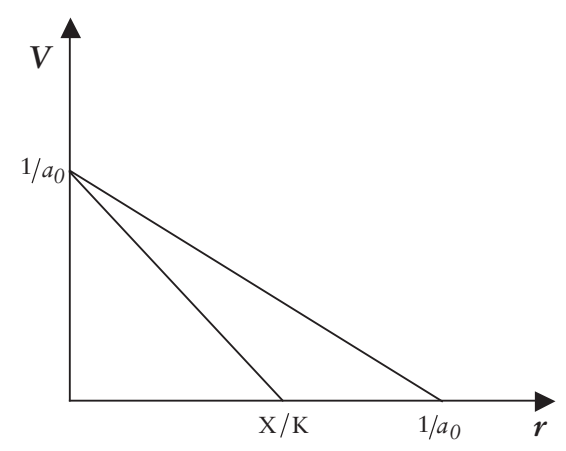


Nessas duas figuras, verifica-se que, quanto menor o grau de utilização da capacidade, mais inclinadas ficam as curvas, com inclinação - $(K / X) / a_{0}$, comparativamente à inclinação $-a_{1} / a_{0}$, observada quando a economia está à plena capacidade.

As equações de preço e produção contêm cinco variáveis, quais sejam, $V, r$, $C, g$ e $K / X$ que medem, respectivamente, a distribuição de renda na economia, o nível de consumo, a taxa de crescimento e o grau de utilização da capacidade produtiva. Precisamos, portanto, de mais três equações para fechar o modelo.

O equilíbrio macroeconômico no mercado de bens implica que:

$$
X=C+I
$$

Admitindo que os trabalhadores gastam toda a sua renda e os capitalistas poupam uma fração constante de seus lucros, denominada $s$, encontramos:

$$
S=I \text { ou } s r K=I
$$

Dividindo-se por $K$, obtemos:

(3) $g=s r$,

a qual afirma que o investimento corresponde à poupança, ambos como fração do estoque de capital.

Se definirmos mais duas hipóteses de comportamento dos agentes, obteremos as equações restantes. Como mencionado anteriormente, essa economia opera em um ambiente predominantemente oligopolístico. Assim sendo, pressupomos que as firmas estabelecem seus preços em cima de custos diretos (custo unitário da mão-de-obra), por meio de uma dada taxa de mark-up, conforme Kalecki. Deste modo, obtemos a seguinte equação de preços da firma:

(4) $\quad P=w a_{0}(1+Z)$

em que $z$ é a taxa de mark-up que depende, dentre outras coisas, do poder monopólico da firma que, por sua vez, depende do grau de concentração industrial.

Uma outra hipótese comportamental refere-se à função investimento. Admitese que o grau de acumulação de capital depende não apenas da taxa de lucro esperada, como também do grau de utilização da capacidade. Por simplificação, admite-se que a taxa de lucro esperada seja igual à corrente. Denominando $u=X / K$, temos que:

(5) $g=g(r, u)$,

com sinal positivo para as duas derivadas parciais, isto é,

$g_{r}>0$ e $g_{u}>0$

É interessante observar que a equação (4) significa que os preços ajustam-se aos custos e são independentes da demanda até a plena ocupação da capacidade. Se, a um dado preço, a demanda for insuficiente para gerar a plena utilização da capacidade, o ajustamento não se dá nos preços, mas sim nas quantidades. Nesse 
sentido, $u<1 / a_{1}$ e, portanto, torna-se natural esperar que a taxa de acumulação seja função também do grau de utilização da capacidade.

Da equação de preços (4) obtém-se o salário real de equilíbrio $V^{*}=\left[a_{0}(1+z)\right]^{-1}$. Substituindo esse valor em (2), temos que:

$$
u=r \frac{(1+z)}{z}
$$

Substituindo essa expressão em (5), obtemos uma relação entre $g$ e $r$ que, combinada com (3), permite determinar $r^{*}$ e $g^{*}$, isto é, a taxa de lucro e a taxa de crescimento de equilíbrio. O grau de utilização da capacidade $\left(u^{*}\right)$ obtém-se pela equação acima, com a substituição de $r^{*}$. Por fim, o consumo por trabalhador $\left(C^{*}\right)$ é obtido substituindo $g^{*}$ em (1). A figura a seguir ilustra a determinação dos valores de equilíbrio de longo prazo.

Figura 3: O modelo de equilíbrio de longo prazo

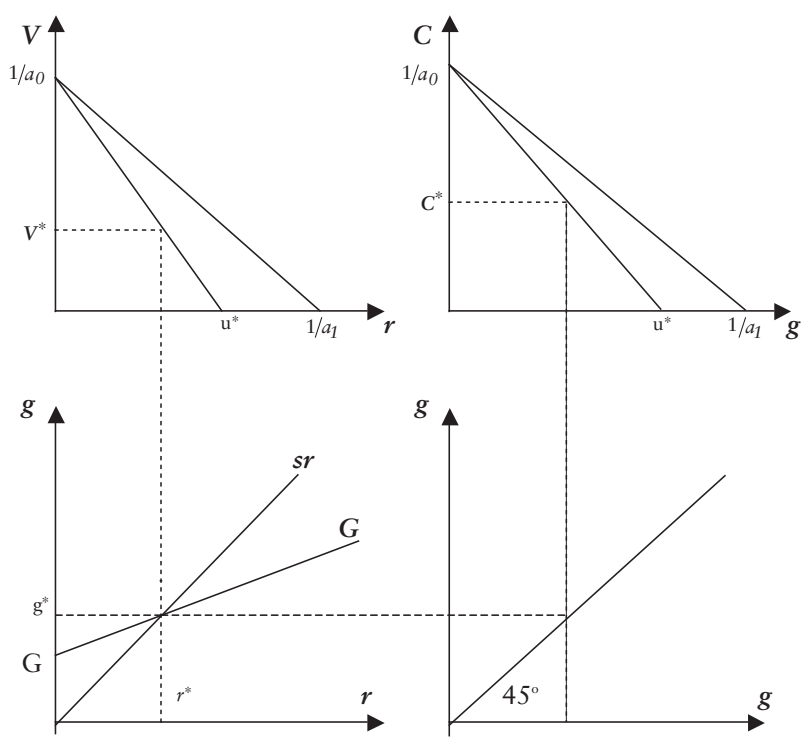

Dos resultados de estática comparativa, o mais interessante é o que ocorre com uma mudança na taxa de mark-up $z$. Uma diminuição em $z$ aumenta o salário real e promove uma redistribuição da renda em favor dos trabalhadores, equação (4). Dada uma queda em $z$, aumenta-se o valor de $V^{*}$. A curva $G G$ torna-se mais inclinada, o que resulta em maiores $g^{*}$ e $r^{*}$, implicando maiores valores de $C^{*}$ e $u^{*}$. Nesse sentido, depreende-se que uma melhoria na distribuição de renda é acompanhada por um aumento na taxa de acumulação de capital, e um salário real mais alto está associado a um aumento na taxa de lucro para uma dada tecnologia. Assim, uma distribuição de renda menos desigual aumenta os gastos de consumo - a demanda agregada - o que provoca aumentos nas taxas de lucro 
no grau de utilização da capacidade e na taxa de acumulação de capital. Esse resultado é conhecido na literatura como estagnacionista, subconsumista ou wageled.

Se a equação (5) tomar a forma linear:

$g=\alpha+\beta r+\gamma u \quad$ com $\quad \alpha>0, \beta>0$ e $\gamma>0$

e notando que $g=s r$ e $r=\pi u,{ }^{6}$ encontramos:

$$
u^{*}=\frac{\alpha}{(s-\beta) \pi-\gamma}
$$

Em termos de estabilidade, assume-se um mecanismo de ajustamento, segundo o qual um excesso de demanda (oferta) gera uma elevação (redução) do produto. Isso equivale à condição de que a função investimento é menos sensível a variações em $u$ que a função poupança (mesma condição que garante a positividade do denominador de $\left.u^{*}\right)$.

Se derivarmos $u$ em relação à margem de lucro $\pi$, teremos o resultado estagnacionista, ou seja,

$$
\frac{d u}{d \pi}=\frac{-\alpha(s-\beta)}{[(s-\beta) \pi-\gamma]^{2}}<0
$$

pois $s>\beta$ (que se segue da condição de estabilidade).

Em outros termos, o modelo apresenta duas características cruciais (Rowthorn, 1982). Em primeiro lugar, tem-se o paradoxo dos custos: qualquer aumento nos custos aumenta a taxa de lucro, ou seja, um aumento no salário real provoca um aumento em $u, r$ e $g$. Por outro lado, um aumento na margem de lucro $\pi$ (queda do salário real) gera numa diminuição em $u$, tornando a curva $G G$ menos inclinada (figura 3 ), implicando uma menor taxa de lucro $r$. Outro paradoxo associado a esse modelo é o da parcimônia. Quanto maior a taxa de poupança $s$, menor a taxa de lucro $r$ e a taxa de acumulação $g$ (para estas conclusões, basta verificar a figura 3).

${ }^{6}$ Seja $\pi$ a participação dos lucros na renda nacional:

(6) $\pi=\frac{r P K}{P X}=1-\frac{w a_{0}}{P}$

Lembrando (4), segue-se que

(6') $\pi=1-\frac{1}{1+z}=\frac{z}{1+z}$

De (2), temos

(7) $\frac{r}{u}=1-\frac{w a_{0}}{P}$

(8) De (6) e (7), segue-se que $\pi=\frac{r}{u}$ ou $r=\pi u$ 


\section{A CRÍTICA DE BHADURI E MARGLIN}

A crítica inicial de Bhaduri e Marglin (1990) refere-se à taxa de lucro como argumento da função investimento, como aparece em Rowthorn (1982), Dutt (1984, 1987, 1990) e Taylor (1985). Como vimos acima (equação 8), a taxa de lucro é igual ao produto da margem de lucro $\pi$ pelo grau de utilização da capacidade $u$. dessa forma, a função investimento é influenciada duas vezes por $u$ (como argumento separado e dentro da taxa de lucro), o que exclui a possibilidade de os capitalistas obterem uma taxa de lucro satisfatória com baixo grau de utilização da capacidade $u$, mas com uma margem de lucro $\pi$ que sobrecompense a queda em $u$ (situação esta que os autores denominam de regime profit-led). A solução encontrada pelos autores é considerar a margem de lucro $\pi$ e o grau de utilização da capacidade $u$ como argumentos separados da função investimento, ou seja:

$$
g=g(\pi, u) \quad g_{\pi}>0 \text { e } g_{u}>0
$$

Ao definir a função investimento na forma acima, os autores tornam possível tanto o resultado estagnacionista (relação positiva entre crescimento e participação dos salários na renda) como o regime profit-led (relação positiva entre crescimento e parcela dos lucros na renda).

$\mathrm{O}$ investimento deve ser igual à poupança para manter o equilíbrio no mercado de bens, i. e.,

$g(\pi, u)=s \pi u$

ou

$F(\pi, u)=g(\pi, u)-s \pi u=0$

A derivada de $u$ em relação a $\pi$ será:

$$
\frac{d u}{d \pi}=-\frac{F_{\pi}}{F_{u}}=\frac{g_{\pi}-s u}{s \pi-g_{u}}
$$

Como o denominador é positivo, - pois assume-se que, na margem, a poupança seja mais sensível a mudanças que o investimento em $u$ para tornar o equilíbrio estável -, então temos que:

$$
\begin{array}{lll}
\frac{d u}{d \pi}<0, \text { se } g_{\pi}-s u<0 & \text { (regime estagnacionista) } \\
\frac{d u}{d \pi}>0, \text { se } g_{\pi}-s u>0 & \text { (regime profit-led) }
\end{array}
$$

Assumindo uma função de investimento linear do tipo:

$$
g=\alpha+\beta \pi+\gamma u,
$$

na qual $\alpha$ pode ser positivo ou negativo, $\beta$ e $\gamma$ positivos. Substituindo $u$ por $r / \pi$, podemos observar que, dada uma queda no mark-up $z$, a curva $G G$ (figura 3 ) fica- 
rá mais inclinada, mas seu intercepto vertical diminuirá, podendo levar ou a valores de $g^{*}$ e $r^{*}$ mais elevados em um regime estagnacionista, ou a valores de $g^{*}$ e $r^{*}$ menores em um regime profit-led.

\section{ECONOMIA ABERTA ${ }^{7}$}

O objetivo desta seção é mostrar as implicações da abertura ao comércio internacional para este tipo de modelo de crescimento e distribuição.

Formalmente, a competitividade internacional pode ser denotada por

$$
\theta=\frac{v P_{f}}{P}
$$

em que $v=$ taxa de câmbio medida em moeda local por unidade de moeda estrangeira, $P_{f}=$ preço dos bens finais dos concorrentes em moeda estrangeira, assumido constante, e $P=$ preço doméstico dos bens finais produzidos localmente.

Diferenciando a equação (9), obtemos o seguinte resultado:

$$
\frac{d \theta}{\theta}=\frac{d v}{v}-\frac{d P}{P}
$$

Dessa forma, à medida em que a desvalorização cambial $d v / v$ superar a taxa de variação de preços domésticos $d P / P$, a competitividade internacional da economia doméstica terá aumentado $d \theta / \theta$. Entretanto, se esse aumento de competitividade irá aumentar ou não o saldo do balanço comercial, dependerá das elasticidades das demandas de exportação e importação em relação aos preços.

O equilíbrio no mercado de bens, em uma economia aberta, deve incluir as exportações $(E)$ e importações $(M)$, qual seja,

$$
\begin{aligned}
& S=I(\pi, u)+E-M \\
& s \pi u=g(\pi, u)+\frac{E-M}{K}
\end{aligned}
$$

Após diferenciar e manipular algebricamente, obtemos ${ }^{8}$

$$
d u=D^{-1}\left(g_{\pi}-s u\right) d \pi+D^{-1} u q \frac{d \theta}{\theta}\left(\eta_{e}+\eta_{m}-1\right)
$$

no qual $D^{-1}=s \pi-g_{u}+\alpha q$ é positivo para atender a condição de estabilidade; $\eta_{e}$ é positivo e representa a elasticidade das exportações em relação à competitividade internacional;

\footnotetext{
${ }^{7}$ Esta seção baseia-se em Bhaduri e Marglin (1990).

${ }^{8}$ Para a demonstração das expressões (11) e (12) a seguir, basta solicitar ao autor.
} 
$\eta_{m}$ é negativo e corresponde à elasticidade das importações em relação à competitividade internacional;

$\alpha$ é positivo e representa a elasticidade das importações em relação ao grau de utilização local;

$q$ corresponde à participação do balanço comercial na renda nacional.

O primeiro termo do lado direito dessa última equação mostra como a desvalorização afeta a utilização da capacidade via mudanças na margem de lucro $\pi$, o que corresponde ao caso de uma economia fechada. O segundo termo refere-se ao efeito do balanço comercial através de alterações na competitividade internacional. Se a desvalorização cambial superar a inflação doméstica (equação 10), a competitividade internacional aumentará, o que resultará num efeito positivo do balanço comercial sobre o grau de utilização da capacidade, desde que a condição Marshall-Lerner seja atendida, ou seja, $\eta_{e}+\eta_{m}-1>0$. Contudo, qual será o impacto da desvalorização cambial sobre a distribuição, em especial sobre o salário real?

Diferenciando a equação da margem de lucro $\pi$ em uma economia aberta, encontramos,

$$
d \pi=(1-\pi)\left[\frac{d P}{P}-\lambda \frac{d w}{w}-(1-\lambda) \frac{d v}{v}\right]
$$

em que $\lambda=\frac{a_{0} w}{a_{0} w+a_{2} P_{f}^{\prime} v}$ e $0<\lambda<1, \alpha_{2}$ matéria-prima importada por unidade do produto, mantida constante, e $P_{f}=$ preço em moeda estrangeira da matéria-prima importada também constante.

Assim, os impactos da desvalorização sobre a competitividade internacional e a margem de lucro são capturados pelas equações (10) e (12). Se a desvalorização aumentar a competitividade internacional $(d \theta>0)$ e a margem de lucro $(d \pi>$ $0)$, então $\frac{d v}{v}>\frac{d P}{P}>\frac{d w}{w} \cdot ?$

A desvalorização, que resulta em menor salário real e maior margem de lucro, aumentará o grau de utilização, estimulando a demanda agregada num regime profit-led, ou seja, o primeiro termo da equação (11) é positivo. O segundo termo será positivo desde que a condição Marshall-Lerner seja satisfeita. Portanto, a estratégia de reduzir o salário real via desvalorização para aumentar a competitividade internacional $(\theta)$ e a margem de lucro $(\pi)$, estimulará o grau de utilização de forma não ambígua no regime profit-led. Por outro lado, num regime estagnacionista, o efeito da desvalorização sobre o grau de utilização é ambíguo. Entretanto, ao observar a eqauação (11), vemos que, quanto maior o grau de

\footnotetext{
$9 \frac{d P}{P}-\lambda \frac{d w}{w}-(1-\lambda) \frac{d v}{v}>0$, o que, após alguma manipulação algébrica, torna-se $\lambda>\left(\frac{d v}{v}-\frac{d P}{P}\right)>\left(\frac{d v}{v}-\frac{d w}{w}\right) \cdot$

Porém, como $0<\lambda<1 \mathrm{e} \frac{d v}{v}-\frac{d w}{w}>0$, para garantir que $d \pi>0$, então:

$\frac{d v}{v}-\frac{d P}{P}<\frac{d v}{v}-\frac{d w}{w}$, ou seja, $\frac{d P}{P}>\frac{d w}{w}$.
} 
abertura da economia indicado por $q$ e maiores as elasticidades $\eta_{e}+\eta_{m}$, mais importante é o efeito do balanço comercial sobre o grau de utilização da capacidade $u$. E, se este efeito dominar quantitativamente o termo negativo na equação (11) num regime estagnacionista, a economia aberta ao comércio internacional assume um caráter profit-led. Em outros termos, o efeito dominante do balanço comercial tende a tornar a lógica estagnacionista crescentemente irrelevante num mundo marcado por alto grau de interdependência externa. Além disso, uma performance exportadora exitosa que mantenha alto o volume de emprego, tende a criar um ambiente de cooperação entre capital e trabalho, apesar do salário unitário real menor. ${ }^{10} \mathrm{O}$ único problema dessa estratégia é a impossibilidade de todos os países alcançarem superávit comercial simultaneamente. ${ }^{11}$

\section{COMENTÁRIOS FINAIS}

Os modelos de crescimento pós-keynesianos introduzem novas considerações na "arte" de modelar o crescimento de uma economia capitalista. A tradição clássica e neoclássica entendem a poupança como o motor de acumulação de capital e assumem que as decisões de poupança sempre conduzem à decisão correspondente de investimento. Nestas últimas, vale a lei de Say e não pode haver nenhuma discrepância entre oferta e demanda agregadas. A introdução de uma função de investimento independente, em conjunto com o grau de utilização da capacidade como uma variável endógena, gera uma nova classe de modelos em que não vale a lei de Say e que abre espaço para resultados de estática comparativa completamente diferentes dos resultados paralelos nos modelos clássico e neoclássico. Mudanças paramétricas como um aumento na propensão a poupar ou na participação dos lucros na renda, que elevam a taxa de acumulação de capital nas economias clássica e neoclássica, podem reduzir a taxa de acumulação de capital nos modelos abordados no presente trabalho. Essas diferenças também levam a diferentes avaliações quanto à política mais apropriada ao crescimento. Sob as perspectivas clássica e neoclássica, uma redistribuição de renda dos lucros para os salários pode ser vista como desejável em si mesma, mas a um preço elevado em termos de menor crescimento. Nos modelos pós-keynesianos atuais, esse trade-off é menos doloroso ou mesmo inexistente: uma redistribuição de renda a favor dos trabalhadores pode mover a economia a um maior grau

\footnotetext{
${ }^{10}$ Para que um regime profit-led seja cooperativo entre capital e trabalho, é necessário que haja um aumento no total de salário real pago, apesar do menor salário real unitário devido ao aumento do volume de emprego e do grau de utilização da capacidade. Em termos formais, requer-se que $\frac{d u \pi}{d \pi u}>\frac{\pi}{1-\pi}$ , ou seja, a elasticidade do grau de utilização em relação à participação dos capitalistas na renda tem de exceder a relação entre as parcelas dos lucros e salários na renda nacional.

${ }^{11}$ Blecker (1989) obteve a mesma conclusão de que a lógica estagnacionista deve ser revista diante da abertura da economia, usando mark-up flexível e o investimento como função da taxa de lucro e do grau de utilização.
} 
de utilização e maior crescimento. Por fim, deve-se observar que esses modelos resgatam a tradição da economia política ao reintroduzir diferentes classes sociais (trabalhadores e capitalistas), o conflito distributivo e a relação de mútua causalidade entre acumulação e distribuição.

\section{REFERÊNCIAS BIBLIOGRÁFICAS}

BERTELLA, M. A. (2000), "O fio da navalha de Harrod e a resposta da escola de Cambridge”, Revista de Análise Econômica, dezembro.

BHADURI, A. e MARGLIN, S. (1990), "Unemployment and the Real Wage: the Economic Basis for Contesting Political Ideologies”, Cambridge Journal of Economics, 14.

BLECKER, R. (1989), “International Competition, Income Distribution, and Economic Growth”, Cambridge Journal of Economics, 13.

DUTT, A. K. (1984), “Stagnation, Income Distribution, and Monopoly Power”, Cambridge Journal of Economics, 8.

DUTT, A. K. (1987), “Alternative Closures Again: a Comment on 'Growth, Distribution and Inflation'”, Cambridge Journal of Economics, 11.

DUTT, A. K. (1990), Growth, Distribution, and Uneven Development, Cambridge University Press.

KALDOR, N. (1956), “Alternative Theories of Distribution”, in Stiglitz, J. E. e Uzawa, H. (1969), Readings in the Modern Theory of Economic Growth, The MIT Press.

LIMA, G. T. (1998), “A Non-Linear Dynamics of Capital Accumulation, Distribution and Conflict Inflation”, in Anais do 26o. Encontro Nacional de Economia, ANPEC, Vitória, dezembro.

LIMA, G. T. (1999), "Progresso tecnológico endógeno, crescimento econômico e distribuição de renda”, in Lima, G. T., Sicsú, J. \& Paula, L. F., Macroeconomia moderna: Keynes e a economia contemporânea, Rio de Janeiro: Campus.

LIMA, G. T. (2000), "Market Concentration and Technological Innovation in a Dynamic Model of Growth and Distribution”, Banca Nazionale del Lavoro Quarterly Review, dezembro.

ROBINSON, J. (1956), The Accumulation of Capital, Macmillan.

ROBINSON, J. (1962), Essays in the Theory of Economic Growth, Macmillan.

ROWTHORN, R. (1982), "Demand, Real Wages and Economic Growth”, in Sawyer, M. C. (1988), Post-Keynesian Economics, Edward Elgar.

STEINDL, J. (1952), Maturity and Stagnation in American Capitalism, Basil Blackwell.

TAYLOR, L. (1985), “A Stagnationist Model of Economic Growth”, Cambridge Journal of Economics, 9 . 